\title{
AVALIAÇÃO DA MADEIRA SERRADA NA AMAZÔNIA MATO-GROSSENSE
}

Assessment of lumber in the Mato-grossense Amazon rainforest

Evaluación de madera aserrada en la Amazonia Mato-grossense

\section{Nathália Toldo Rissi ${ }^{1}$, Rafael Rodolfo de Melo*2, Allyson Rocha Alves ${ }^{2}$, Felipe Gomes} Batista $^{3}$, Maila Janaína Coelho de Souza ${ }^{3}$, Lucélio Mendes Ferreira

${ }^{1}$ Universidade Federal de Mato Grosso - UFMT, Instituto de Ciências Agrárias e Ambientais, Sinop, Brasil.

${ }^{2}$ Universidade Federal Rural do Semi-Árido - UFERSA, Departamento de Ciências Agronômicas e Florestais, Mossoró, Brasil.

${ }^{3}$ Universidade Federal do Rio Grande do Norte - UFRN, Programa de Pós-Graduação em Ciências Florestais, Macaíba, Brasil.

*Correspondência: Departamento de Ciências Agronômicas e Florestais, Av. Francisco Mota, 572 - Bairro Costa e Silva, Mossoró RN | CEP: 59.625-900,e-mail rafael.melo@ufersa.edu.br

Artigo recebido em 11/08/2019 aprovado em 07/11/2020 publicado em 04/03/2021.

\section{RESUMO}

O presente trabalho teve como objetivo avaliar a madeira serrada na Amazônia mato-grossense. $\mathrm{O}$ estudo ocorreu por meio de um levantamento realizado em 37 serrarias da região Norte de Mato Grosso, abrangendo as cidades de Sinop, Sorriso, Feliz Natal, Aripuanã, Juara, Tabaporã, e União do Sul. Foram avaliadas as padronizações das peças serradas com as dimensões estabelecidas pelas normas da Associação Brasileira de Normas Técnicas (ABNT); e verificada se as principais espécies serradas na região são identificadas corretamente, por meio de comparações entres as amostras comercializadas com dados de xilotecas credenciadas. Foi analisada qual a espécie que ocorre com maior abrangência nas serrarias da região é a Qualea paraensis. As peças mais produzidas foram caibros, vigas e tábuas. Destes, as tábuas foram os produtos em que as serrarias mais seguem as normas com as dimensões recomendadas. Das principais espécies comercializadas na região, verificou que pelo menos $30 \%$ são comercializadas com nomenclatura equivocada.

Palavras-chave: Espécies tropicais, Amazônia Legal, Padronização.

\section{ABSTRACT}

The objective of this study was to evaluate the quality of sawn wood in the Mato-grossense Amazon region. The study was carried in 37 sawmills in the Northern region of Mato Grosso, including the cities of Sinop, Sorriso, Feliz Natal, Aripuanã, Juara, Tabaporã and União do Sul. The standardization of the sawed pieces with the dimensions established by the standards of the Brazilian Association of Technical Standards (ABNT) was evaluated; and verified if the main species sawed in the region are identified correctly, through comparisons among samples sold with data from accredited stores. Qualea paraensis was analyzed which species occurs most widely in sawmills of the region. The most produced pieces were rafters, beams and boards. Of these, the boards were the products in which the sawmills most followed the standards with the recommended dimensions. Of the main species traded in the region, it was verified that at least $30 \%$ of these species are identified/marketed under the wrong nomenclature.

Keywords: Tropical species, Amazon Forest, Standardization.

\section{RESUMEM}

El presente estudio tuvo como objetivo evaluar la madera aserrada en la Amazonía de Mato Grosso. El estudio se llevó a cabo mediante una encuesta realizada en 37 aserraderos de la región norte de Mato Grosso, que abarcó las ciudades de Sinop, Sorriso, Feliz Natal, Aripuanã, Juara, Tabaporã y União do Sul. dimensiones establecidas por los estándares de la Asociación Brasileña de Normas Técnicas (ABNT); y verificó si las principales especies aserradas de la región están correctamente identificadas, mediante comparaciones entre las muestras comercializadas con datos de xilotecas acreditadas. Qualea paraensis fue analizada como la especie que más se 
presenta en los aserraderos de la región. Las piezas más producidas fueron vigas, vigas y tablas. De estos, los tableros fueron los productos en los que los aserraderos más siguen las normas con las dimensiones recomendadas. De las principales especies comercializadas en la región, se encontró que al menos el 30\% se comercializa con la nomenclatura incorrecta.

Descriptores: Especies tropicales, Amazonia legal, Normalización.

\section{INTRODUÇÃO}

A extração madeireira no estado de Mato Grosso teve início nos anos sessenta, em função das políticas de integração nacional que objetivavam a incorporação de suas áreas às atividades produtivas. Com a extração da cobertura florestal para futuras instalações de áreas de pecuária e agricultura, muitas empresas madeireiras foram instaladas na região para processamento destas árvores. $\mathrm{Na}$ época, com a abundância de matéria-prima, havia pouca ou nenhuma preocupação com os aspectos qualitativos do material explorado (Melo et al., 2017; Melo et al., 2019). Como reflexo desse comportamento, os principais setores que dependem dessa produção, como a construção civil e a indústria moveleira, se tornaram cada vez mais críticos quanto à qualidade da madeira produzida na Região. Tais críticas se baseavam principalmente na padronização das dimensões, na correta identificação e na falta de informações técnicas para uma melhor indicação de uso destas espécies (Rissi, 2015).

A padronização das peças, além de permitir uma maior qualidade, possibilita ainda uma série de outras vantagens, entre as quais: a confiança do usuário nos elementos normalizados, a permutabilidade dos produtos utilizados, maior facilidade e presteza dos trabalhos realizados (Mascaró, 1975). No Brasil, a padronização das bitolas de madeira serrada se dá pela Norma Brasileira Regulamentadora (NBR) elaborada pela Associação Brasileira de Normas Técnicas (ABNT). As padronizações para madeira serrada podem ser observadas nas seguintes normativas: NBR 7203 (ABNT, 1982); NBR 7190 (ABNT, 1997); NBR 14807 (ABNT, 2002); e a Resolução CONAMA
474/2016 (Brasil, 2016). Nestas podem ser encontradas dimensões comerciais e nomenclaturas para as diferentes peças serradas.

Além das dimensões das pelas, a identificação científica correta das espécies é fundamental para o desenvolvimento das ciências básica e aplicada, bem como para garantir a integridade das transações comerciais e qualidade da madeira. Nas serrarias, muitas espécies são conhecidas por diferentes nomes vulgares o que dificulta realmente a sua identificação. Não existe uma padronização entre as nomenclaturas vernacular e científica. Uma espécie pode receber diversos nomes vernaculares, bem como várias espécies podem ser designadas por um único nome (Martins-da-Silva et al., 2003).

$\mathrm{O}$ agrupamento de várias espécies em um único nome torna inviável a correta definição de seu uso, pois cada espécie madeireira tem suas características peculiares (como secagem, densidade, trabalhabilidade, contração, flexão, compressão, dureza, tração e cisalhamento) que define não somente seu uso final como também o processo de beneficiamento para comercialização (Procópio e Secco, 2008). A utilização adequada das espécies de madeira depende de procedimentos que garantam a identificação destas, quer seja como árvores, toras ou madeira serrada (Kennan e Tejada, 1984).

Diante disso, torna-se necessário estudar a qualidade da madeira serrada do norte de Mato Grosso, visando à melhoria da qualidade do produto para os setores que são dependentes do mesmo como a construção civil e indústria moveleira. O presente trabalho tem como objetivo avaliar alguns aspectos da madeira serrada na Amazônia mato-grossense. Para isso, serão avaliadas as dimensões da madeira serrada 
nas serrarias da região e verificadas se estas se encontram de acordo com a norma vigente da ABNT. Além disso, será verificada se as espécies comercializadas na região são identificadas corretamente e, comercializadas de forma adequada.

\section{MATERIAIS E MÉTODOS}

\section{Área de estudo e coleta de dados}

O estudo foi realizado em diferentes serrarias localizadas na Amazônia mato-grossense, mais precisamente na região do Norte de Mato Grosso, abrangendo as cidades de Sinop, Sorriso, Feliz Natal, Aripuanã, Juara, Tabaporã, e União do Sul. Foram realizadas visitas em trinta e sete (37) serrarias de pequeno e médio porte, onde foram coletadas informações por meio de um questionário. Foram obtidas informações relativas às espécies utilizadas, as peças produzidas por cada espécie e as dimensões de cada produto. Em seguida, foram coletadas amostras das espécies que estavam disponíveis no pátio de cada serraria e etiquetadas conforme nome comum e, sempre que possível, o nome científico fornecido pelo proprietário.

\section{Identificação das espécies}

As amostras etiquetadas foram enviadas para a Xiloteca do Laboratório de Produtos Florestais pertencente ao Serviço Florestal Brasileiro (LPF-SFB) em Brasília para correta identificação de cada uma das amostras e possível confrontação com as informações apresentadas pelos proprietários. As peças selecionadas foram comparadas com as amostraspadrão da Xiloteca. Com base nessa comparação e, com o auxílio da análise de um especialista do Laboratório, foram feitas as identificações de cada uma das amostras coletadas. Quando não foi possível identificar em nível de espécie, identificou-se em nível de gênero.

O preparo das amostras para identificação foi realizado seguindo as recomendações propostas por Zaque e Melo (2019). Inicialmente as amostras foram preparadas no seu plano transversal (topo), deixando uma superfície bem polida e plana, obtida com auxílio de uma lâmina afiada. Em seguida, utilizou-se para as observações uma lupa tipo conta fios com aumento de 10x, que apresenta foco fixo, permitindo comparações. Toda análise foi realizada em local com boa iluminação. Posteriormente, foi feito um leve umedecimento na superfície polida, para realçar os caracteres macroscópicos a serem investigados.

\section{Avaliação das dimensões das peças serradas}

Com a entrevista realizada nas serrarias foi obtido às dimensões e a nomenclatura utilizada para cada peça produzida. Estas informações, seguindo as recomendações de Oliveira et al. (2008), foram comparadas com as dimensões e nomenclaturas propostas pela ABNT em suas normativas (ABNT, 1982; ABNT, 1997; ABNT, 2002) (Tabela 1).

Tabela 1. Nomenclaturas e dimensões das normas da ABNT utilizada para comparação.

\begin{tabular}{clll}
\hline \multirow{2}{*}{ Nomenclatura da peça } & \multicolumn{3}{c}{ Dimensões - Esp. / Larg. (cm) } \\
\cline { 2 - 4 } & NBR 7203 & NBR 7190 & NBR 14807 \\
\hline Caibro & $4,0-8,0 / 5,0-8,0$ & $2,5 /$ NE & $4,0-8,0 / 5,0-8,0$ \\
Prancha & $4,0-7,0 />20,0$ & Não especificado & $3,9-7,0 />16,1$ \\
Pranchão & $>7,0 />20,0$ & Não especificado & $7,1-16,1 />16,1$ \\
Ripa & $<2,0 /<10,0$ & Não especificado & $1,0-2,0 / 2,0-5,0$ \\
Sarrafo & $2,0-4,0 / 2,0-10,0$ & $2,5 /$ NE & $2,1-3,9 / 2,0-9,9$ \\
Tábua & $1,0-4,0 />10,0$ & Não especificado & $1,0-3,7 />10,0$ \\
Viga & $>4,0 / 11,0-20,0$ & $5 /$ NE & $4,0-8,0 / 8,1-16,0$ \\
Vigota & $4,0-8,0 \times 8,0-11,0$ & $5 /$ NE & Não especificado
\end{tabular}




\section{RESULTADOS E DISCUSSÃO}

\section{Espécies madeireiras utilizadas na região}

Em levantamentos realizados sobre o perfil do setor de base florestal na Amazônia Legal, consta que de um total de 3.000 espécies madeireiras já identificadas há o aproveitamento industrial de apenas 230. O pequeno número de espécies florestais comerciais explorada comercialmente, intensifica a busca por algumas espécies, o que proporciona subaproveitamento dessas áreas (Barbosa et al, 2001).
Foi identificado que a espécies Qualea paraensis, é a que tem maior representatividade no setor madeireiro nessa região, isso se deve, pois a espécie tem suas características conhecidas, assim se torna de fácil uso e de fácil trabalhabilidade (Tabela 2). Estudos desenvolvido por Melo et al. (2015) na mesma região do estudo, também foi verificada a maior frequência desta espécie nos planos de manejo florestais.

Tabela 2: Nome comum, nome científico e percentual de serrarias que desdobram cada uma das

espécies utilizadas.

\begin{tabular}{|c|c|c|}
\hline Nome Comum & Nome científico $*$ & Serrarias $(\%)$ \\
\hline Amescla/Morcegueira & Trattinickia burseraefolia (Mart.) Wild & 27,5 \\
\hline Angelim Pedra & Hymenolobium modestum Ducke & 32,5 \\
\hline Angelim Saia & Parkia pendula (Willd.) Benth. ex Walp & 5,0 \\
\hline Angelim/Angelim Amargoso & Vatairea sp. & 10,0 \\
\hline Breu/ Amescla aroeira & Trattinickia sp. & 5,0 \\
\hline Cambará & Qualea paraensis & 70,0 \\
\hline Canelão & Ocotea $\mathrm{sp}$ & 30,0 \\
\hline Caroba & Jacaranda copaia (Aubl.) D. Don & 2,5 \\
\hline Catuaba/ Maria preta & Qualea sp. & 7,5 \\
\hline Cedrinho & Erisma uncinatum Warm. & 55,0 \\
\hline Cedro & Cedrela sp. & 22,5 \\
\hline Cedro Amazonense & Cedrela odorata L. & 2,5 \\
\hline Cedro Boleiro & Juniperus sp. & 2,5 \\
\hline Cedro Rosa & Cedrela fissilis & 5,0 \\
\hline Champanhe/Cumaru & Dipteryx odorata (Aubl.) Wild & 40,0 \\
\hline Copaíba & Copaifera multijuga Hayne & 2,5 \\
\hline Cupiúba/ Peróba Fedida/ Vinagreiro & Golpia glabra Aubl. & 35,0 \\
\hline Escova de Macaco & Apeiba echinata Gaertn & 2,5 \\
\hline Faveira & Dinizia excelsa Ducke & 2,5 \\
\hline Garapeira & Apuleia leiocarpa & 37,5 \\
\hline Guanandi & Calophyllum brasiliensis Cambess. & 2,5 \\
\hline Guaritá & Astronium sp. & 5,0 \\
\hline Itaúba & Mezilaurus itauba (Meisn.) Taub.Ex Mez & 37,5 \\
\hline Jatobá & Hymenaea courbaril $\mathrm{L}$. & 15,0 \\
\hline Jequitibá & Cariniana sp. & 5,0 \\
\hline Leiteiro & Brosimum lactescens (S. Moore) C. C. Berg & 2,5 \\
\hline Marupá & Simarouba amara & 2,5 \\
\hline Mirindiba & Buchenavia sp. & 2,5 \\
\hline Oiticica & Clarisa racemosa & 7,5 \\
\hline Paricá & Schizolobium amazonicum & 2,5 \\
\hline Peróba & Aspidosperma sandwithianum Markgr. & 15,0 \\
\hline Peróba d'água & Aspidosperma pachypterum Müll Arg & 2,5 \\
\hline Peróba Mica/ Peróba Amarela & Aspidosperma polyneuron & 7,5 \\
\hline Sucupira Amarela & Enterolobium schomburgkii (Benth.) Benth. & 7,5 \\
\hline Sucupira Preta & Diplotropis sp. & 12,5 \\
\hline Tachi & Sclerolobium sp. & 2,5 \\
\hline Tamboril & Enterolobium contortisiliquum & 2,5 \\
\hline Tatajuba & Chlorophora tinctoria & 7,5 \\
\hline Tauari & Couratari sp. & 10,0 \\
\hline Uxirana & Coиepia paraenses & 2,5 \\
\hline
\end{tabular}


*provável nome científico obtido por meio de consulta a material bibliográfico considerando as espécies ocorrentes na região, conforme citação do nome vulgar feito pelos produtores.

A espécie Qualea paraensis é uma das dez (10) espécies que tem maior presença no comércio na região, outras espécies abundantes comercialmente são Golpia glabra (Cupiúba), Erisma uncinatum (Cedrinho), Mezilaurus itauba (Itaúba), Hymenolobium sp (Angelim), Apuleia sp (Garapeira), Manilkara sp (Maçaranduba), Cordia goeldiana (Freijó), Dipteryx sp (Cumbarú), Trattinickia sp (Amescla) (Ribeiro, 2013), sendo que das espécies (gêneros) citadas(os) sete (7) estão com representatividade alta na Tabela 2.

Pode perceber que 15 espécies apresentaram pouca presença nas serrarias, com apenas $2,5 \%$, isso acontece, pois as espécies não estão disponíveis em grande quantidade para as serrarias ou não apresentam características adequadas para seu uso. A espécie Calophyllum brasiliensis Cambess é considerada madeira "de lei", é uma espécie rara e está em extinção, isso confirma a sua baixa representatividade nas serrarias (Duffecky e Fossati, 2009).

\section{Nomenclaturas e dimensões das peças serradas}

Apesar das normativas estabelecidas pela ABNT proporem apenas oito diferentes nomenclaturas para as peças de madeira serrada (caibro; prancha; pranchão; ripa; sarrafo; tábua; viga; e vigota), ao todo foram observadas nas serrarias da região 15 nomenclaturas diferentes para as peças de madeira serrada obtidas nas serrarias, ou seja, 07 a mais do que estabelecida pela ABNT. Tal diversidade dificulta a comercialização de peças de madeira pela impossibilidade de seus usuários identificarem com facilidades as possíveis peças de madeira. Adicionalmente, o uso da madeira como material de construção fica em desvantagem, principalmente quando comparadas aos seus tradicionais concorrentes do setor, que geralmente apresentam nomenclatura em dimensões normatizadas e claramente definidas.

Além das nomenclaturas propostas pela ABNT, foram observados os seguintes produtos serrados: caixaria; meia cana; quadrado; ripão; régua; cabinho e pé direito. Além dos produtos serrados foram encontrados produtos beneficiados como assoalho; cobertura; decker; forro; madeira a vista; rodapé e vista de porta. A presença dos produtos beneficiados deve-se a características de algumas serrarias da região, apresentar anexo a sua unidade de beneficiamento primário de madeiras, uma unidade de beneficiamento de madeira. Estes resultados estão de acordo com a afirmação feita por Rocha (1999), que no caso do Brasil, muitas vezes pode-se encontrar anexadas à serraria, ou mesmo no seu interior, unidades de beneficiamento, ou seja, não são unidades de desdobro (primário/secundário) e sim de usinagem de madeiras. Os produtos beneficiados se querem são mencionados na $\mathrm{ABNT}$, sendo assim há necessidade de normas quanto aos produtos beneficiados, que são diferentes dos serrados.

Os principais produtos da indústria de madeira são madeiras serradas, incluindo madeira para construção, assoalhos, laminados, compensados e móveis (Reis, 1989). No Tabela 3 podem ser observadas as nomenclaturas de peças que são produzidas na região com as suas respectivas dimensões e que não constam na ABNT.

Tabela 3. Peças serradas que não estão estabelecidas nas normas com as dimensões que são utilizadas.

\begin{tabular}{cc}
\hline Peças & Dimensões \\
\hline Caixaria & $2,0-2,5 \times 15,0-30,0$ \\
Meia cana & $2,5 \times 2,0-5,0$ \\
Quadrado & $10,0>\times 10,0>$ \\
Ripão & $2,0 \times 15,0 ; 2,5 \times 5,0$ \\
Régua & $3,0 \times 15,0$ \\
Cabinho & $2,0 \times 2,0$ \\
Pé direito & $15,0 \times 15,0 ; 20,0 \times 20,0 ; 30,0 \times 30,0$ \\
\hline
\end{tabular}


Na Tabela 4 podem ser observadas as peças produzidas para cada uma das espécies nas diferentes serrarias avaliadas. Tais resultados nos auxiliam a verificar quais as espécies apresentam uma maior versatilidade de uso, sendo utilizadas para produção das mais diferenciadas tipologias de produtos, assim como, as de uso mais restrito. As espécies que apresentaram maior versatilidade para produção de peças serradas e de beneficiamento são: Trattinickia burseraefolia, Qualea paraenses, Ocotea sp, Erisma uncinatum, Cedrela sp., Dipteryx odorata, Golpia glabra e Mezilaurus itauba. Essas espécies citadas são mais versáteis em relação a sua diversidade de uso, além de apresentarem uma disponibilidade no comércio. Tais resultados corroboram com aqueles apresentados na Tabela 2.

Tabela 4. Produtos de madeira para cada uma das espécies serradas.

\begin{tabular}{|c|c|c|c|c|c|c|c|c|c|}
\hline \multirow{2}{*}{ Espécies utilizadas } & \multicolumn{9}{|c|}{ Produtos } \\
\hline & $\mathbf{C a}$ & Pr & Pro & $\mathbf{R i}$ & $\mathbf{S a}$ & Tá & $\mathbf{V i}$ & Vo & $\mathbf{N E}$ \\
\hline Trattinickia burseraefolia (Mart.) Wild & $\mathrm{x}$ & $\mathrm{x}$ & $\mathrm{x}$ & $\mathrm{x}$ & $\mathrm{x}$ & $\mathrm{x}$ & $\mathrm{x}$ & $\mathrm{x}$ & $\mathrm{x}$ \\
\hline Hymenolobium modestum Ducke & $\mathrm{x}$ & $\mathrm{x}$ & $\mathrm{x}$ & $\mathrm{x}$ & $\mathrm{x}$ & $\mathrm{x}$ & $\mathrm{x}$ & $\mathrm{x}$ & \\
\hline Parkia pendula (Willd.) Benth. ex Walp & $\mathrm{x}$ & & & & & $\mathrm{x}$ & & & $\mathrm{x}$ \\
\hline Vatairea sp. & $\mathrm{x}$ & $\mathrm{x}$ & & & & $\mathrm{x}$ & $\mathrm{x}$ & $\mathrm{x}$ & \\
\hline Trattinickia sp. & $\mathrm{x}$ & & & & & & $\mathrm{x}$ & $\mathrm{x}$ & \\
\hline Qualea paraenses Ducke & $\mathrm{x}$ & $\mathrm{x}$ & $\mathrm{x}$ & $\mathrm{x}$ & $\mathrm{x}$ & $\mathrm{x}$ & $\mathrm{x}$ & $\mathrm{x}$ & $\mathrm{x}$ \\
\hline Ocotea $\mathrm{sp}$ & $\mathrm{x}$ & $\mathrm{x}$ & $\mathrm{x}$ & $\mathrm{x}$ & $\mathrm{x}$ & $\mathrm{x}$ & $\mathrm{x}$ & $\mathrm{x}$ & $\mathrm{x}$ \\
\hline Jacaranda copaia (Aubl.) D. Don & $\mathrm{x}$ & & & & & $\mathrm{x}$ & & & \\
\hline Qualea sp. & $\mathrm{x}$ & $\mathrm{x}$ & & $\mathrm{X}$ & $\mathrm{x}$ & $\mathrm{x}$ & $\mathrm{x}$ & $\mathrm{x}$ & \\
\hline Erisma uncinatum Warm. & $\mathrm{x}$ & $\mathrm{x}$ & $\mathrm{x}$ & $\mathrm{x}$ & $\mathrm{x}$ & $\mathrm{x}$ & $\mathrm{x}$ & $\mathrm{x}$ & $\mathrm{x}$ \\
\hline Cedrela sp. & $\mathrm{x}$ & $\mathrm{x}$ & $\mathrm{x}$ & $\mathrm{x}$ & $\mathrm{x}$ & $\mathrm{x}$ & $\mathrm{x}$ & $\mathrm{x}$ & $\mathrm{x}$ \\
\hline Cedrela odorata L. & & $\mathrm{x}$ & & & & $\mathrm{x}$ & & & \\
\hline Juniperus sp & $\mathrm{x}$ & & & $\mathrm{X}$ & $\mathrm{x}$ & & $\mathrm{x}$ & $\mathrm{x}$ & \\
\hline Cedrela fissilis & & & & & & & & & $\mathrm{x}$ \\
\hline Dipteryx odorata (Aubl.) Wild. & $\mathrm{x}$ & $\mathrm{x}$ & $\mathrm{x}$ & $\mathrm{X}$ & $\mathrm{x}$ & $\mathrm{x}$ & $\mathrm{x}$ & $\mathrm{x}$ & $\mathrm{x}$ \\
\hline Copaifera multijuga Hayne & $\mathrm{x}$ & & & & & $\mathrm{x}$ & & & \\
\hline Golpia glabra Aubl. & $\mathrm{x}$ & $\mathrm{x}$ & $\mathrm{x}$ & $\mathrm{X}$ & $\mathrm{x}$ & $\mathrm{x}$ & $\mathrm{X}$ & $\mathrm{x}$ & $\mathrm{x}$ \\
\hline Apeiba echinata Gaertn & $\mathrm{x}$ & & & & & $\mathrm{x}$ & & & \\
\hline Dinizia excelsa Ducke & $\mathrm{x}$ & $\mathrm{x}$ & & $\mathrm{x}$ & & $\mathrm{x}$ & $\mathrm{x}$ & $\mathrm{x}$ & $\mathrm{x}$ \\
\hline Apuleia leiocarpa & $\mathrm{x}$ & $\mathrm{x}$ & $\mathrm{x}$ & $\mathrm{x}$ & $\mathrm{x}$ & $\mathrm{x}$ & $\mathrm{x}$ & $\mathrm{x}$ & \\
\hline Calophylum brasiliensis Cambess. & & & & & & & & & $\mathrm{x}$ \\
\hline Astronium sp. & & $\mathrm{x}$ & & & & $\mathrm{x}$ & & & \\
\hline Mezilaurus itauba (Meisn.) Taub. ex Mez & $\mathrm{x}$ & $\mathrm{x}$ & $\mathrm{x}$ & $\mathrm{x}$ & $\mathrm{x}$ & $\mathrm{x}$ & $\mathrm{x}$ & $\mathrm{x}$ & $\mathrm{x}$ \\
\hline Hymenaea courbaril L. & & $\mathrm{x}$ & $\mathrm{x}$ & $\mathrm{x}$ & & $\mathrm{x}$ & $\mathrm{x}$ & & $\mathrm{x}$ \\
\hline Cariniana sp. & & $\mathrm{x}$ & & & & & $\mathrm{x}$ & & \\
\hline Brosimum lactescens (S. Moore) C. C. Berg & $\mathrm{x}$ & & & & & & $\mathrm{x}$ & $\mathrm{x}$ & \\
\hline Simarouba amara & & & & & & & & & $\mathrm{x}$ \\
\hline Buchenavia sp. & $\mathrm{x}$ & & & $\mathrm{X}$ & & & $\mathrm{x}$ & $\mathrm{x}$ & \\
\hline Clarisa racemosa & $\mathrm{x}$ & $\mathrm{x}$ & & & & & $\mathrm{x}$ & $\mathrm{x}$ & \\
\hline Scchizolobium amazonicum & $\mathrm{x}$ & & & & & & $\mathrm{x}$ & $\mathrm{x}$ & \\
\hline Aspidosperma pachypterum Müll Arg & $\mathrm{x}$ & & & & & & $\mathrm{x}$ & & \\
\hline Aspidosperma polyneuron & & & & & & & $\mathrm{x}$ & $\mathrm{x}$ & $\mathrm{X}$ \\
\hline Enterolobium schomburgkii (Benth.) Benth. & $\mathrm{x}$ & $\mathrm{x}$ & & $\mathrm{x}$ & $\mathrm{x}$ & $\mathrm{x}$ & $\mathrm{x}$ & $\mathrm{x}$ & \\
\hline Diplotropis sp. & $\mathrm{x}$ & $\mathrm{x}$ & $\mathrm{x}$ & & $\mathrm{x}$ & $\mathrm{x}$ & $\mathrm{x}$ & $\mathrm{x}$ & $\mathrm{x}$ \\
\hline Sclerolobium sp. & & & & & $\mathrm{x}$ & $\mathrm{x}$ & & & \\
\hline Enterolobium contortisiliquum & & & & & & $\mathrm{x}$ & & & \\
\hline Chlorophora tinctoria & & & & & & & & & $\mathrm{x}$ \\
\hline Couratari sp. & & $\mathrm{x}$ & & & & & $\mathrm{x}$ & & \\
\hline Couepia paraenses & $\mathrm{x}$ & & & & & & $\mathrm{x}$ & $\mathrm{x}$ & \\
\hline
\end{tabular}

Legenda: Ca: caibro; Pr: prancha; Pro: pranchão; Ri: Ripa; Sa: sarrafo; Tá: tábua; Vi: viga; Vo: vigota; NE: não especificado nas normas.

Jankowski (1990) confirma que a espécie, Erisma uncinatum é indicada para o uso de móveis populares e armações de móveis, embalagens leves, compensados, molduras, guarnições, rodapés, cabos 
de vassouras, forros, lambris, formas para concreto e tábuas em geral. A Mezilaurus itauba vem sendo largamente usada em construções externas, como postes, moirões, dormentes, cruzetas e estacas; em partes internas, utilizada também como vigas, caibros, marcos ou batentes de portas e janelas, esquadrias e tacos, isto porque a madeira é muito pesada e dura, com alta resistência mecânica e elevada resistência natural ao apodrecimento e ao ataque de insetos, por isso possui maior versatilidade (Garcia et al., 2012).

Já as espécies que apresentam produtos somente beneficiados e que não constam na ABNT foi observada para Calophylum brasiliensis, Simarouba amara, Cedrela fissilis e Chlorophora tinctoria.

O Tabela 5 apresenta o percentual de serrarias que possui cada produto estabelecido nas normas. Entre as peças mais produzidas na região estão o caibro com $81,08 \%$ e a viga com $86,48 \%$. Estas peças apresentam pequenas dimensões e são produzidas com as partes das toras que não há possibilidades de produzir produtos maiores, como é o caso do pranchão que apresenta baixa representatividade com apenas $59,46 \%$. A vigota foi o produto que apresentou menor percentual, com apenas 54,05\%.

Tabela 5. Avaliação da quantidade de serrarias que seguem as normas das dimensões das peças serradas.

\begin{tabular}{ccc}
\hline \multirow{2}{*}{ Produto } & \multicolumn{2}{c}{ Serrarias que tem o produto } \\
\cline { 2 - 3 } & Número & $\mathbf{( \% )}$ \\
\hline Caibro & 30 & 81,08 \\
Prancha & 23 & 62,16 \\
Pranchão & 22 & 59,46 \\
Ripa & 28 & 75,67 \\
Sarrafo & 23 & 62,16 \\
Tábua & 30 & 81,08 \\
Viga & 32 & 86,48 \\
Vigota & 20 & 54,05 \\
\hline
\end{tabular}

Com relação à adequabilidade das normas para as diferentes peças produzidas, analisando a Tabela 6 é possível verificar que a tábua foi o produto em que as serrarias mais seguem as normas com 86,67\% na NBR 7203 e com 83,33\% na NBR 14807. $\mathrm{O}$ produto que obteve menor percentual foi o sarrafo na NBR $7190-17,39 \%$.

Tabela 6. Avaliação do percentual de serrarias que sequem as Normas

\begin{tabular}{crrr}
\hline \multirow{2}{*}{ Produto } & \multicolumn{3}{c}{ Serrarias que seguem as Normas (\%) } \\
\cline { 2 - 4 } & NBR 7203 & NBR 7190 & NBR 14807 \\
\hline Caibro & 70,00 & 0,00 & 70,00 \\
Prancha & 52,17 & NE & 30,44 \\
Pranchão & 63,64 & NE & 18,18 \\
Ripa & 50,00 & NE & 25 \\
Sarrafo & 30,43 & 17,39 & 17,40 \\
Tábua & 86,67 & NE & 83,33 \\
Viga & 68,75 & 37,50 & 34,37 \\
Vigota & 20,00 & 35,00 & NE \\
\hline
\end{tabular}

Legenda: NE- não especificado.

Apesar de uma quantidade significativa de serrarias seguirem as normas, durante a visita os madeireiros admitiram trabalharem com "bica corrida", ou seja, eles fabricam os produtos de acordo com o pedido do cliente. Estes justificam a prática como forma de atender as demandas de clientes para ter uma maior competitividade. A falta de fiscalização se as empresas estão seguindo ou não as normas, também colabora com esse cenário. Além disso, a disponibilidade de normas diferentes também dificulta a padronização, já que todas as normas apresentadas se encontram vigente.

\section{Identificação das espécies}

Das 21 espécies encaminhadas para identificação de especialistas, observou que $66,66 \%$ apresentavam conformidade com a descrição apresentadas pelos produtores. Consequentemente, observou-se que as demais espécies encaminhadas $(33,33 \%)$ foram identificadas por especialistas como 
espécies diferentes das mencionadas pelas serrarias (Tabela 7).

Outro caso que pode ser citado é a identificação apenas com o nome vulgar, como já dito, uma espécie pode apresentar diversos nomes vulgares. Foi o que aconteceu com a espécie Jacaranda copaia que foi nomeada por vulgarmente Caroba na região do estudo (Mato Grosso) e, é conhecida por Pará-Pará na região onde foi realizada a análise de laboratório (Brasília-DF), sendo a nomenclatura científica a mesma para ambas.

A identificação incorreta das espécies tem pontos negativos, não apenas para a fiscalização de órgãos competentes, como para os consumidores que podem estar adquirindo um produto que seria de uma qualidade maior ou menor, desvalorizando assim o valor pago. Ressalta-se, ainda, que espécies determinadas cientificamente valorizam o nome da empresa que as comercializa, garantem competitividade em mercados de maior excelência e agregam valor aos produtos comercializados.

Outro fator que poderia ser melhorado pelas instituições seria a pesquisa para conhecer as propriedades físicas e mecânicas das diversas espécies que a Amazônia possui, para que haja um maior aproveitamento e uma redução no uso e/ou procura excessiva por algumas espécies, o que pode vir a acarretar uma significativa redução destas nas matas da região, podendo levar até a extinção pelo uso predatório e/ou manejo inadequado.

Tabela 7. Comparação entre a identificação das espécies disponibilidade pelos produtores nas serrarias com a correta identificação das amostras feitas em xiloteca.

\begin{tabular}{|c|c|c|}
\hline Serrarias* & Xiloteca & Verificação \\
\hline Trattinickia burseraefolia (Mart.) Wild & Trattinnickia sp. & Adequada \\
\hline Hymenolobium modestum Ducke & Erisma sp. & Inadequada \\
\hline Parkia sp. & Parkia pendula & Adequada \\
\hline Vatairea sp. & Qualea sp. & Inadequada \\
\hline Qualea paraensis & Qualea sp. & Adequada \\
\hline Ocotea $\mathrm{sp}$ & Ocotea $\mathrm{sp}$. & Adequada \\
\hline Erisma uncinatum Warm. & Erisma sp. & Adequada \\
\hline Cedrela sp. & Erisma sp. & Inadequada \\
\hline Juniperus sp. & Vochysia sp. & Inadequada \\
\hline Dipteryx odorata (Aubl.) Wild & Dipteryx sp. & Adequada \\
\hline Copaifera multijuga Hayne & Copaifera sp. & Adequada \\
\hline Golpia glabra Aubl. & Golpia glabra & Adequada \\
\hline Apeiba echinata Gaertn & Apeiba echinata & Adequada \\
\hline Apuleia leiocarpa & Golpia glabra & Inadequada \\
\hline Mezilaurus itauba (Meisn.) Taub.Ex Mez & Mezilaurus sp. & Adequada \\
\hline Hymenaea courbaril $\mathrm{L}$. & Hymenaea sp. & Adequada \\
\hline Aspidosperma sandwithianum Markgr. & Golpia glabra & Inadequada \\
\hline Aspidosperma polyneuron & Aspidosperma sp. & Adequada \\
\hline Golpia glabra Aubl. & Caraipa sp. & Inadequada \\
\hline Diplotropis sp. & Diplotropis sp. & Adequada \\
\hline Jacaranda copaia (Aubl.) D. Don & Jacaranda copaia & Adequada \\
\hline
\end{tabular}

*De acordo com o apresentado no Tabela 2.

\section{CONCLUSÃO}

Foram observadas 21 diferentes espécies desdobradas pelas serrarias na Amazônia matogrossense (considerando a identificação correta das espécies realizadas pela xiloteca). Todavia, quando essa diversidade é confrontada com o número de espécies que cada serraria trabalha, uma espécie se destaca das demais - o cambará (Qualea paraensis), presente em aproximadamente $70 \%$ das serrarias avaliadas.

Parte considerável das empresas da região não segue nenhuma normativa a respeito do 
dimensionamento e nomenclatura de peças de madeira. Foram observados diversos produtos comercializados com nomes e dimensões diferentes dos apresentados pelas normas vigentes. Parte significativa das serrarias visitadas adota o sistema de "bica corrida", que é o dimensionamento de peças de acordo com as exigências do comprador. Apesar disso, há interesse dos produtores por uma padronização do setor, desde que haja uma fiscalização para que os padrões sejam adotados por todas as serrarias, para que nenhuma empresa seja beneficiada por oferecer produtos diferenciados (por não seguirem as normas).

Deste modo, uma maior divulgação das normas para o conhecimento dos consumidores, além dos comerciantes, também é essencial nesse processo.

As serrarias da região estudada necessitam de melhorias no processo de qualidade dos produtos, devendo ser levado em consideração à correta identificação das espécies utilizadas e a adequações as normas que estabelecem o dimensionamento de peças de madeira serradas.

Todos os autores declararam não haver qualquer potencial conflito de interesses referente a este artigo.

\section{REFERÊNCIAS}

ASSOCIAÇÃO BRASILEIRA DE NORMAS TÉCNICAS - ABNT. NBR 14807/2002: Peças de madeira serrada: dimensões. Rio de Janeiro: ABNT, 2002.

ASSOCIAÇÃO BRASILEIRA DE NORMAS TÉCNICAS - ABNT. NBR 7190/1997: Projeto de estruturas de madeira. Rio de Janeiro: ABNT, 1997.

ASSOCIAÇÃO BRASILEIRA DE NORMAS TÉCNICAS - ABNT. NBR 7203/1982: Madeira serrada e beneficiada. Rio de Janeiro: ABNT, 1982.

BARBOSA, A. P.; VIANEZ, B. F.; VAREJÃO, M. J.; ABREU, R. L. S. Considerações sobre o perfil tecnológico do setor madeireiro na Amazônia Central. Parcerias Estratégicas, v. 12, n. 1, p. 42- 61, 2001.

BRASIL. CONSELHO NACIONAL DE MEIO AMBIENTE - CONAMA. Resolução CONAMA
474/2016: Dispõe sobre procedimentos para inspeção de indústrias consumidoras ou transformadoras de produtos e subprodutos florestais madeireiros de origem nativa. Brasília: CONAMA, 2016.

DUFFECKY, M. D.; FOSSATI, L. C. Avaliação da adaptação de Calophyllum brasiliensis cambess. (guanandi), família Clusiaceae, no planalto Norte Catarinense. Ágora: Revista de Divulgação Científica, v. 16, n. 2, p. 14-27, 2009.

GARCIA, F. M.; MANFIO, D. R.; SANSIGOLO, C. A.; ANGELI, C.; MAGALHÃES, P. A. D. Rendimento no desdobro de toras de Itaúba (Mezilaurus itauba) e Tauari (Couratari guianensis) segundo a classificação da qualidade da tora. Floresta e Ambiente, v. 19, n. 4, p. 468-474, 2012.

KEENAN, F. J.; TEJADA, M. Tropical timber for building materials in the Andean group countries of South America. Ontario: International Development Research Centre-IDRC; 1984.

MARTINS-DA-SILVA, R. C.V.; HOPKINS, M. G.; THOMPSON, I. S. Identificação botânica na Amazônia: situação atual e perspectivas. Belém: Embrapa; 2003. 77p.

MASCARÓ, L. A. R. Caracterização do setor produtivo de materiais de construção. São Paulo: Universidade de São Paulo; 1975.

MELO, R. R.; DACROCE, J. F.; RODOLFO JR, F.; LISBOA, G. S.; FRANCA, L. C. J. Lumber Yield of Four Native Forest Species of the Amazon Region. Floresta e Ambiente, v. 26, n. 1, p. 1-7, 2019.

MELO, R. R.; ROCHA, M. J.; RODOLFO JR, F.; STANGERLIN, D. M. Análise da influência do diâmetro no rendimento em madeira serrada de cambará (Qualea sp.). Pesquisa Florestal Brasileira, v. 36, n. 88, p. 393, 2017.

MELO R. R.; PASTORE K. C.; MASCARENHAS A. R. P.; ACOSTA, F. C.; PEDROSA, T. D.; SERENINI JR, L. Vouchers for releasing forestry credit (CLCF) for Sinop, Mato Grosso, Brazil. Nativa, v. 3, n. 1, p. 36-43, 2015.

OLIVEIRA, V. M.; DEL MENEZZI, C. H. S.; CAMARGOS, J. A. A.; VALE, A. T. Adequação às normas e qualidade da madeira serrada para fins estruturais comercializada no Distrito Federal. Floresta, v. 38, n. 3, p. 405-412, 2008.

PROCÓPIO, L. C.; SECCO. R. S. A importância da identificação botânica nos inventários florestais: o exemplo do "tauari" (Couratari spp. e Cariniana spp. 
- Lecythidaceae) em duas áreas manejadas no estado do Pará. Acta Amazonica, v. 38, n. 1, p. 31-44, 2008.

REIS, M. S. Mesa Redonda internacional, oportunidade e limitações para o desenvolvimento da indústria baseada em madeiras tropicais na América Latina. Brasília: IBDF; 1989.

RIBEIRO, E. S. Comercialização de madeira serrada de florestas naturais em mato grosso: um diagnóstico do setor de base florestal. Dissertação de Mestrado. Universidade Federal de Mato Grosso - UFMT; 2013.
RISSI, N. T. Avaliação da qualidade da madeira serrada no Estado de Mato Grosso. Monografia de Graduação, Universidade Federal de Mato Grosso UFMT; 2015.

ROCHA, M. P. Desdobro primário da madeira. Curitiba: UFPR; 1999.

ZAQUE, L. A. M.; MELO, R. R. Caracterização macroscópica de madeiras da Amazônia. Pará de Minas: Virtual Books; 2019. 114p. 